\title{
Pathology and Protein Changes of the Spinal Dural Arteriovenous Fistula Arterial Draining Vein Under Sustained High Vascular Pressure
}

\begin{abstract}
Peixi Liu ${ }^{1,2,3,4+}$, Yuan Shi ${ }^{1,2,3,4 t}$, Sichen Li ${ }^{1,2,3,4}$, Yingjun Liu ${ }^{1,2,3,4}$, Yingjie Zhou ${ }^{5}$, Yaying Song ${ }^{6}$, Wei Zhu ${ }^{1,2,3,4 *}$ and Qingzhu An ${ }^{1,2,3,4 *}$

${ }^{1}$ Department of Neurosurgery, Huashan Hospital of Fudan University, Shanghai, China, ${ }^{2}$ Neurosurgical Institute of Fudan University. Shanghai, China, ${ }^{3}$ Shanghai Clinical Medical Center of Neurosurgery, Shanghai, China, ${ }^{4}$ Shanghai Key Laboratory of Brain Function and Restoration and Neural Regeneration, Shanghai, China, ${ }^{5}$ Department of Hand Surgery, Huashan Hospital, Fudan University, Shanghai, China, ${ }^{6}$ Department of Neurology, Renji Hospital, School of Medicine, Shanghai Jiao Tong University, Shanghai, China
\end{abstract}

Object: Spinal dural arteriovenous fistula (SDAVF) is the most common spinal vascular shunt lesion. Although pathological changes in the SDAVF draining vein (SDAVF-DV) have been elucidated, protein changes remain enigmatic. We investigated the pathology and protein changes in the SDAVF-DV under sustained high vascular pressure.

Methods: Three SDAVF-DV samples were compared with superficial temporal artery (STA) and superficial temporal vein (STV) samples as controls. Vascular structure was revealed by hematoxylin and eosin (H\&E) and Masson staining; and cell distribution, extracellular matrix, and inflammation infiltration were observed by immunohistochemistry. Label-free quantitative proteomics was performed, and the peptide mixture was fractionated and analyzed by liquid chromatography-tandem mass spectrometry (LC-MS/MS) to identify differentially expressed proteins. Bioinformatics analysis of the differentially expressed proteins was performed using Gene Ontology (GO), Kyoto Encyclopedia of Genes and Genomes (KEGG), and protein-protein interaction (PPI) networks.

Results: H\&E and Masson staining showed an artery-like structure of the SDAVF-DV. Immunostaining showed that VWF+ cells were not continuous in the SDAVF-DV. Although $\alpha-S M A+$ and AT1 + cells were more abundant in the STV than in the SDAVF-DV, piezo-1 expression was lower in the SDAVF-DV. The SDAVF-DV showed different distributions of elastin, COL I, and COL III. COL IV and COL VI were decreased in the SDAVF-DV, while CD45+ cells and COX-1 were increased compared with those in the controls. No differences in CD68 expression and COX-2 staining were observed between the SDAVF-DV and controls. Compared with the STA, 95 proteins were upregulated and 303 proteins were downregulated in the SDAVF-DV. The most differential GO terms in each category were the adenylate cyclase-modulating G protein-coupled receptor signaling pathway, U6 snRNP, and SH3 domain binding. The most differentially expressed KEGG protein pathway was focal adhesion. Compared with the STV, the SDAVF-DV had 158 upregulated proteins and 362 downregulated proteins. The most differential GO terms in each category were lamellipodium assembly, U6 snRNP, and 
SH3 domain binding; and the most differentially expressed KEGG protein pathway was dilated cardiomyopathy. PPI analysis revealed PPIs among the top 300 proteins.

Conclusions: The SDAVF-DV exhibits specific pathology and protein expression changes under sustained high vascular pressure. The results of the present study provide insights into the pathogenesis of SDAVF formation at the protein level as well as a scientific foundation for further exploration of the pathophysiological mechanism of the SDAVF.

\section{Keywords: SDAVF draining vein, superficial temporal artery, superficial temporal vein, pathology, proteomics}

\section{INTRODUCTION}

Spinal dural arteriovenous fistula (SDAVF) is the most common spinal vascular shunt lesion characterized by an abnormal connection between a radicular meningeal artery and a radicular

Abbreviations: SDAVF, spinal dural arteriovenous fistula; SDAVF-DV, spinal dural arteriovenous fistula draining vein; STA, superficial temporal artery; STV, superficial temporal vein; KEGG, Kyoto Encyclopedia of Genes and Genomes. medullary vein. As venous connections drain to radicular veins, the draining vein shows gradual arterialization. Because of venous hypertension, clinical presentations and progressive myelopathy can be assessed.

In SDAVF, venous drainage is provided by longitudinal spinal veins linked together and to the epidural network $(1,2)$. In many clinical case reports, the arterialized SDAVF draining vein (SDAVF-DV) was identified easily after opening the dura during an operation. The pathology of the arterialized SDAVF-DV

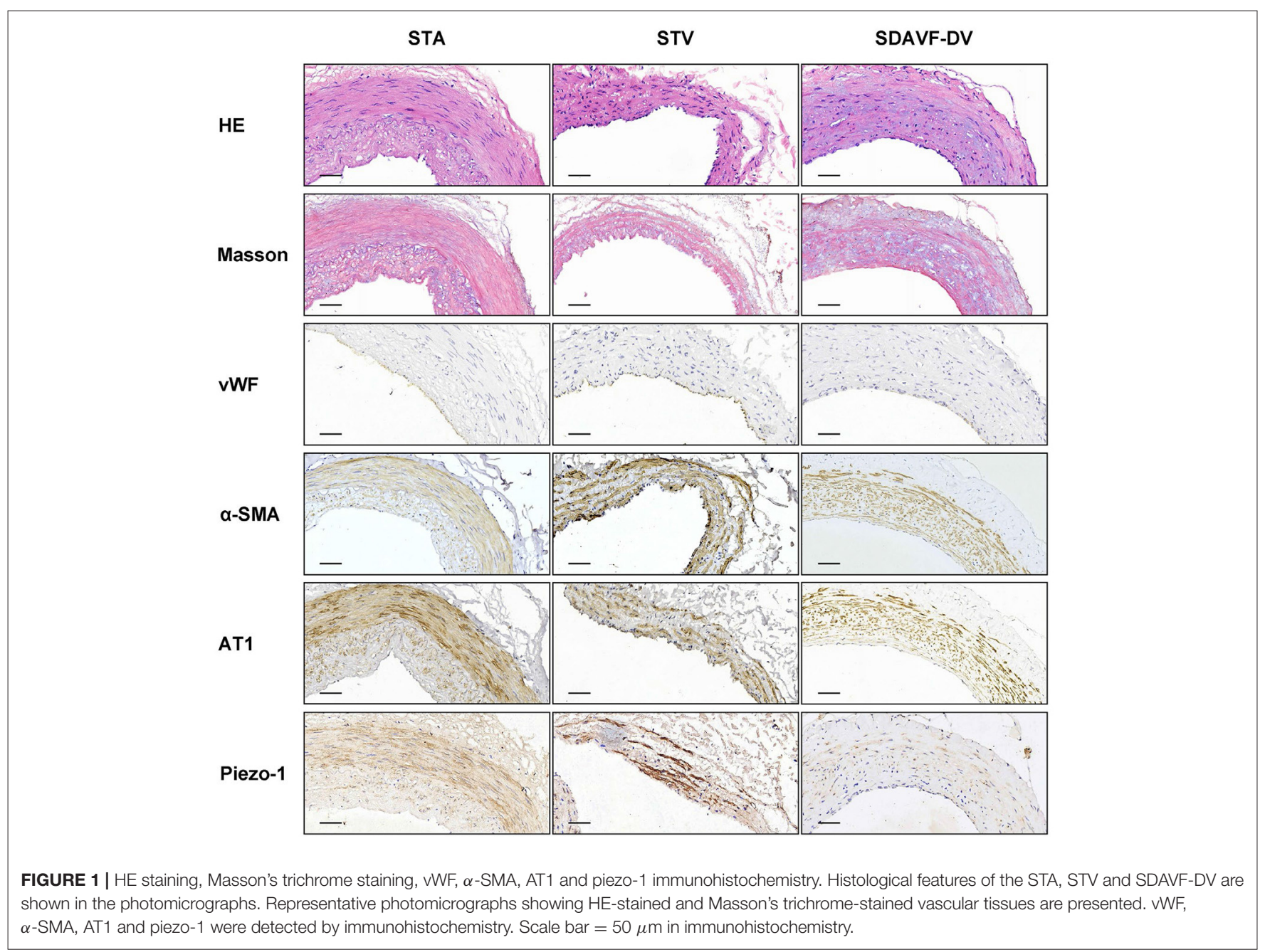



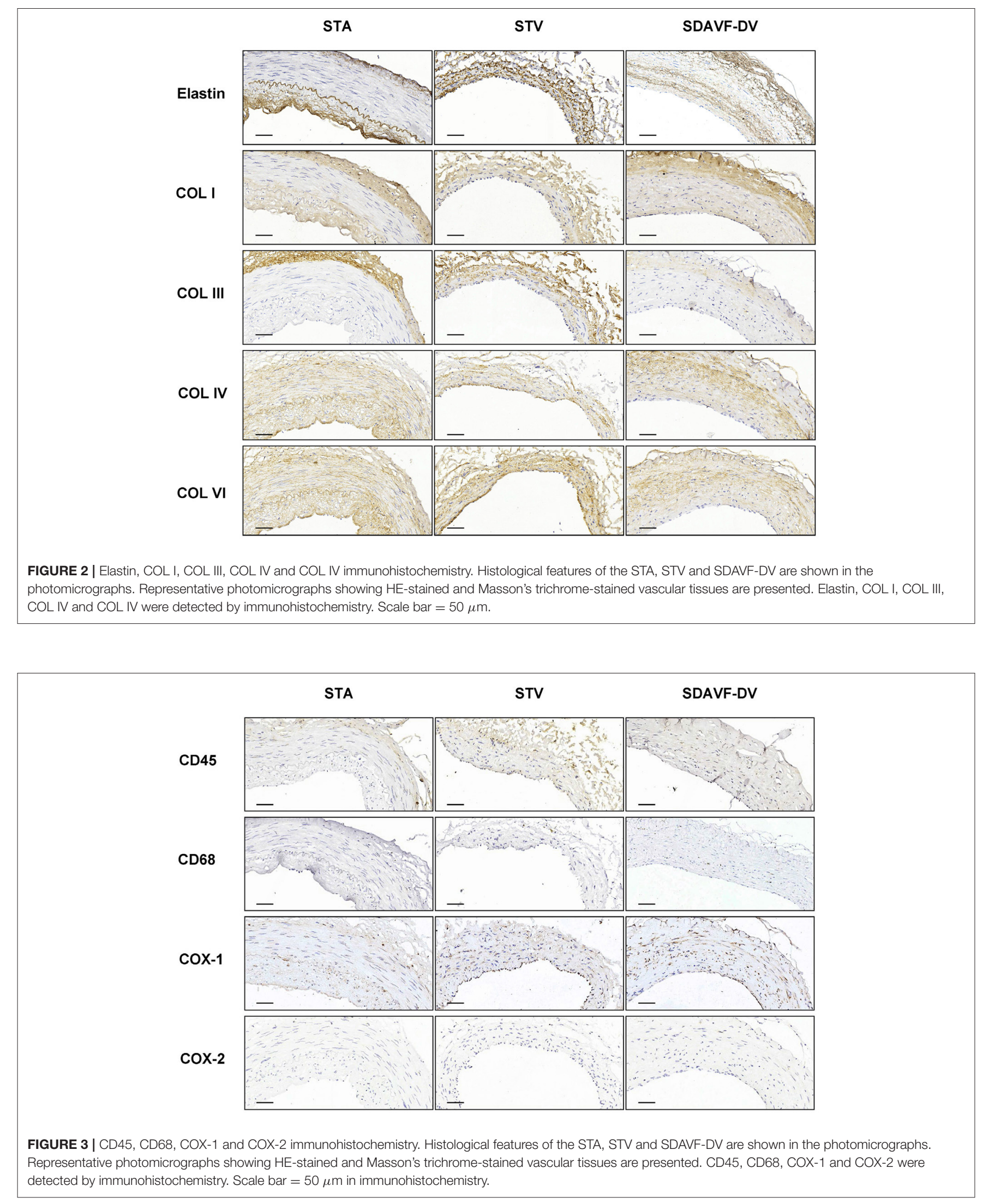
was mentioned in a previous study. However, the protein changes in this arterialized vein under high intravascular pressure remain enigmatic.

In the present study, we used quantitative proteomics to compare the SDAVF-DV with the superficial temporal artery (STA) and superficial temporal vein (STV) to show different protein expression levels under venous hypertension. The results of our present study might provide insights into the pathogenesis of SDAVF formation at the protein level.

\section{METHODS}

\section{Ethics Statement}

The current study was examined and approved by the Ethics Committee of Huashan Hospital, Fudan University.
Each participant provided their written informed consent to participate in this study.

\section{Patients and Tissue Sample Preparation}

Three SDAVF-DVs were removed after microsurgery ligation. Three STAs and three STVs were obtained from patients with intracranial tumors via the extended pterional approach $(3,4)$. We used the samples from each group for the comparative proteomics analysis. The tissues used for the proteomics analysis were immediately frozen in liquid nitrogen and stored at $-80^{\circ} \mathrm{C}$. The SDAVF-DVs, STAs, and STVs were homogenized in a $4 \%$ sodium dodecyl sulfate (SDS), $100 \mathrm{mM}$ of Tris- $\mathrm{HCl}$, and $100 \mathrm{mM}$ of DTT solution. Then, a fluorescence assay was conducted to determine the total protein concentration. Approximately 200 $\mu \mathrm{g}$ of total protein from the tissues was proteolysed on a 10 $\mathrm{kDa}$ filter (PALL Life Sciences, Shanghai, China) using a Filter

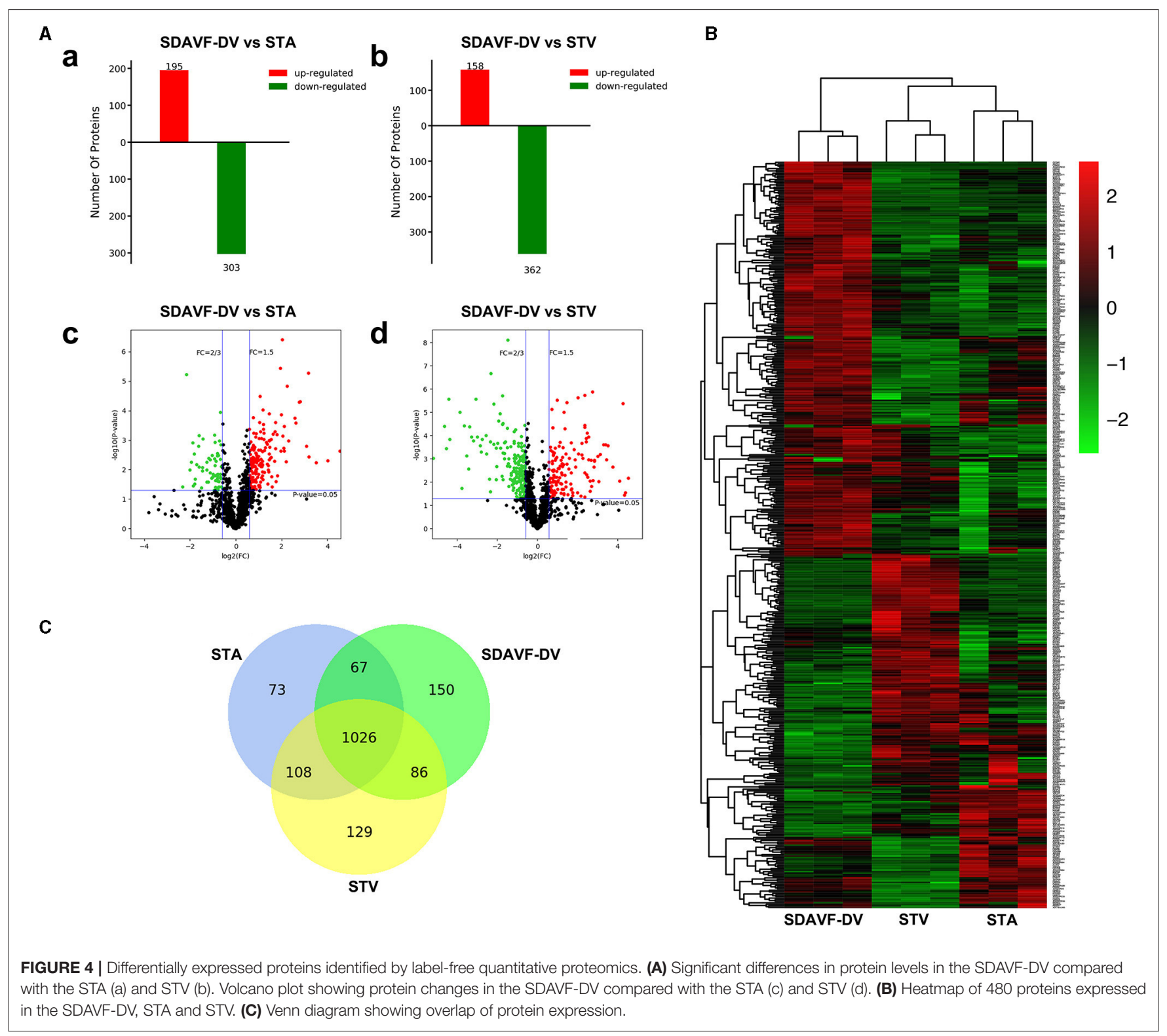


TABLE 1 | Top $10 \mathrm{GO}$ term (SDAVF-DV vs STA).

Category Top 10 upregulated GO term $\begin{aligned} & \text { Top } 10 \text { downregulated GO } \\ & \text { term }\end{aligned}$

term

Biological process

adenylate cyclase-modulating

translation reinitiation

G-protein coupled receptor

signaling pathway

lamellipodium assembly

lamellipodium morphogenesis

regulation of epithelial cell

migration

regulation of microtubule-based

process

wound healing

regulation of receptor recycling

ESCRT III complex disassembly

regulation of osteoclast

differentiation

trophectodermal cell

differentiation

Cellular component

$\begin{array}{ll}\begin{array}{l}\text { nuclear pore outer ring } \\ \text { GATOR2 complex }\end{array} & \text { U6 snRNP } \\ \text { mitochondrial envelope } & \text { Lsm1-7-Pat1 complex } \\ \text { elastic fiber } & \text { ESCRT III complex } \\ & \begin{array}{l}\text { cytoplasmic side of plasma } \\ \text { membrane }\end{array} \\ \text { GAIT complex } & \text { membrane coat } \\ \text { intermediate filament } & \text { endoplasmic reticulum exit site } \\ \text { neurofilament cytoskeleton } & \text { nuclear outer membrane } \\ \text { microfibril } & \text { rough endoplasmic reticulum } \\ \text { membrane raft } & \text { LINC complex }\end{array}$

Molecular function

SH3 domain binding

antioxidant activity

ATP-dependent NAD(P)H-hydrate dehydratase activity

ADP-dependent

$\mathrm{NAD}(\mathrm{P}) \mathrm{H}$-hydrate dehydratase

activity

phosphatidylinositol

phospholipase $\mathrm{C}$ activity

phospholipase $\mathrm{C}$ activity

metallopeptidase activity

G-protein coupled serotonin

receptor binding

3-hydroxyacyl-CoA

dehydrogenase activity

acetyl-CoA C-acyltransferase

activity
IRES-dependent translational initiation

ribosome disassembly

regulation of growth

regulation of cardiac muscle hypertrophy

positive regulation of calcineurin-NFAT signaling cascade

plasma membrane repair

regulation of vascular endothelia growth factor receptor signaling pathway

prostaglandin biosynthetic

process

secretion

smooth muscle contractile fiber

phospholipid binding

protein disulfide oxidoreductase activity

lyase activity

glutathione binding

prostaglandin-E synthase activity

catalytic activity

protein serine/threonine phosphatase inhibitor activity

peroxidase activity

interleukin-1 receptor antagonist activity

proteinase activated receptor binding
Aided Sample Preparation (FASP) protocol as described in detail elsewhere (5). The peptide solution was transferred to a solidphase extraction cartridge (Empore $7 \mathrm{~mm} / 3 \mathrm{ml}$ ) for desalting and cleanup. The peptide samples were resuspended in water with $0.1 \%$ formic acid (v/v), and the protein content was estimated by UV light spectral density at $280 \mathrm{~nm}$ (6) prior to analysis by nano-liquid chromatography-tandem mass spectrometry $(\mathrm{N}-$ LC-MS/MS).

\section{$H$ and $E$ Staining, Masson Staining, and Immunostaining}

Sections were randomly chosen from each sample for hematoxylin and eosin staining (H\&E), Masson's trichrome staining (Sigma, St. Louis, MO, USA), and immunohistochemistry. Anti-von Willebrand factor (vWF) (Abcam, Cambridge, MA, USA), anti- $\alpha$-smooth muscle actin ( $\alpha$-SMA) (Invitrogen, Carlsbad, CA, USA), anti-angiotensin receptor 1 (AT1) (Abcam), anti-piezo-1 (Novus Biologicals, Cambridge, UK), anti-elastin (Abcam), anti-collagen I (COL I) (Abcam), anti-collagen III (COL III) (Abcam), anti-collagen IV (COL IV) (Abcam), anti-collagen VI (COL VI) (Abcam), antiCD45 (Abcam), anti-CD68 (Abcam), anti-COX-1 (Cell Signaling Technology, Danvers, MA, USA), and anti-COX-2 (Abcam) were used as the primary antibodies. 3,3/-Diaminobenzidine (DAB) plus chromogen (Thermo Fisher Scientific, Waltham, MA, USA) was used for substrate visualization according to the manufacturer's protocol.

\section{Label-Free Quantitative Analysis and Data Processing}

Trypsin-digested peptides from the tissues were analyzed by LC-MS/MS; each sample was analyzed twice. All raw Xcalibur files acquired from the MS runs were analyzed using the default settings of MaxQuant software (version 1.3.0.5) with minor modifications as previously described (7). Hierarchical clustering was performed with MEV software (v4.6, TIGR). The differentially expressed proteins $(p<0.05)$ were analyzed by hierarchical clustering to identify potential markers capable of classifying all samples.

The clustering pattern, expression analyses, and volcano plots were based on R software according to standardized data. Venn diagrams of the characteristics of each of the three groups of differentially expressed proteins were generated.

The Gene Ontology (GO) and enrichment analyses of the dysregulated proteins in this experiment were based on the publicly available databases DAVID 6.7 (http://david.abcc.ncifcrf. gov/) and QuickGO (http://www.ebi.ac.uk/QuickGO/).

The genomic, chemical, and systemic functions of the dysregulated proteins were analyzed and enriched by Kyoto Encyclopedia of Genes and Genomes (KEGG) analysis (http://www.kegg.jp/kegg/pathway.html). The significance of differential protein enrichment in each pathway entry was calculated using the hypergeometric distribution test and is expressed as the $p$-value. Predicted protein-protein interaction (PPI) networks for these differentially expressed proteins were 
TABLE 2 | Top 10 GO term (SDAVF-DV vs STV).

Category Top 10 upregulated GO term $\begin{aligned} & \text { Top } 10 \text { downregulated GO } \\ & \text { term }\end{aligned}$

Biological process

lamellipodium assembly

lamellipodium morphogenesis

neuromuscular synaptic

transmission

dGTP catabolic process

regulation of innate immune

response

dATP catabolic process

regulation of receptor recycling

ESCRT III complex disassembly

positive regulation of epithelial to mesenchymal transition

positive regulation of glycogen

biosynthetic process

\section{Cellular component}

zonula adherens
cell-substrate adherens junction
sarcoplasm
mitochondrial small ribosomal
subunit
mitochondrial oxoglutarate
dehydrogenase complex
mitochondrial envelope
phosphopyruvate hydratase
complex
alphav-
beta3 integrin vitronectin complex
cytosolic ribosome
microtubule minus-end
function
SH3 domain binding
activity
activity
activity
dGTP binding
insulin receptor binding
long-Coyity
activity

exonucleolytic

nuclear-transcribed mRNA catabolic process involved in deadenylation-dependent decay

ubiquitin homeostasis

endosomal vesicle fusion

positive regulation of pinocytosis regulation of cardiac muscle hypertrophy

positive regulation of calcineurin-NFAT signaling cascade

early endosome to Golgi transport

regulation of cardiac muscle contraction

protein targeting to plasma membrane

positive regulation of voltage-gated calcium channel activity

U6 snRNP

Lsm1-7-Pat1 complex

Cajal body

macropinosome

ciliary pocket membrane

ESCRT III complex

cytoplasmic side of plasma membrane

membrane coat

smooth muscle contractile fiber integrin alpha8-beta1 complex

Molecular function

phospholipase A2 activator activity

PDZ domain binding

phosphate ion binding

phosphatidylinositol phosphate binding

phospholipid binding

cysteine-type peptidase activity

catalytic activity

peroxidase activity

interleukin-1 receptor antagonist activity

muscle alpha-actinin binding constructed using the STRING database (http://string.embl.de/) and Cytoscape software (http://www.cytoscape.org/).

\section{Statistical Analysis}

The statistical analysis was performed with IBM SPSS, and the graphs were generated with GraphPad Prism software. The significance of differences between two groups in the proteomics analysis was assessed using one-way analysis of variance (ANOVA). Proteins were defined as significantly differentially expressed when the ratio was $\geq 2$ or $\leq 0.5$ in the SDAVF-DV compared with normal tissues $(p<0.01)$.

\section{RESULTS}

\section{Differences in Pathology Among the Spinal Dural Arteriovenous Fistula Draining Vein, Superficial Temporal Artery, and Superficial Temporal Vein}

H\&E and Masson staining showed an artery-like structure of the SDAVF-DV. Immunostaining revealed that $\mathrm{vWF}+$ cells were not continuous. $\alpha$-SMA+ and AT1 $1+$ cells showed an increase in smooth muscle cells, and the cell distribution was disordered in the SDAVF-DV. Piezo-1 expression was lower in the SDAVF-DV tunica media (Figure 1). The distributions of elastin, COL I, and COL III was different in the SDAVF-DV. COL IV and COL VI were decreased in the SDAVF-DV (Figure 2). The SDAVF-DV exhibited more CD45+ cells and COX-1 expression than did the controls. No differences in CD68 expression and COX-2 staining were observed between the SDAVF-DV and controls (Figure 3).

\section{Identification of Differentially Expressed Proteins in the Spinal Dural Arteriovenous Fistula Draining Veins, Superficial Temporal Arteries, and Superficial Temporal Veins} Three paired SDAVF-DV, STA, and STV tissue samples were analyzed in the initial discovery phase.

An equal amount of protein from each tissue was digested. Then, the peptides were analyzed by N-LC-MS/MS. Using MaxQuant (version 1.3.0.5), we identified 2,829 nonredundant proteins with a local false discovery rate (FDR) $<1 \%$ and at least two unique peptides per protein. The label-free quantification (LFQ) intensity ratios for the 2,829 proteins were calculated, and significant differences in the protein expression levels between two tissues were determined using a $t$-test $(p<0.05)$. Compared with the STA, the SDAVF-DV had 195 significantly upregulated proteins and 303 significantly downregulated proteins. Compared with the STV, among the 520 proteins that exhibited significant differences, 158 were significantly upregulated and 362 were significantly downregulated in the SDAVF-DV (Figure 4A). When the three groups were combined, 480 differentially expressed proteins were identified (shown in the heatmap in Figure 4B). Venn analysis showed the variation and commonalities of different proteins in each group. A total of 1,026 proteins were expressed in all groups, and 150 proteins were identified only in the SDAVF-DV (Figure 4C). 


\section{Gene Ontology Analysis}

We performed GO analysis to analyze the differentially expressed proteins. When comparing the SDAVF-DV with the STA, most of differential GO terms expressed in each category were the adenylate cyclase-modulating $G$ protein-coupled receptor signaling pathway, U6 snRNP, and SH3 domain binding. We examined the top 10 upregulated and downregulated GO terms in the biological process, cellular component, and molecular function categories with 2.0-fold $(p<0.05)$ differential gene expression (Table 1).

\section{A}

\section{Gene Ontology Classification (SDAVF-DV vs STA)}

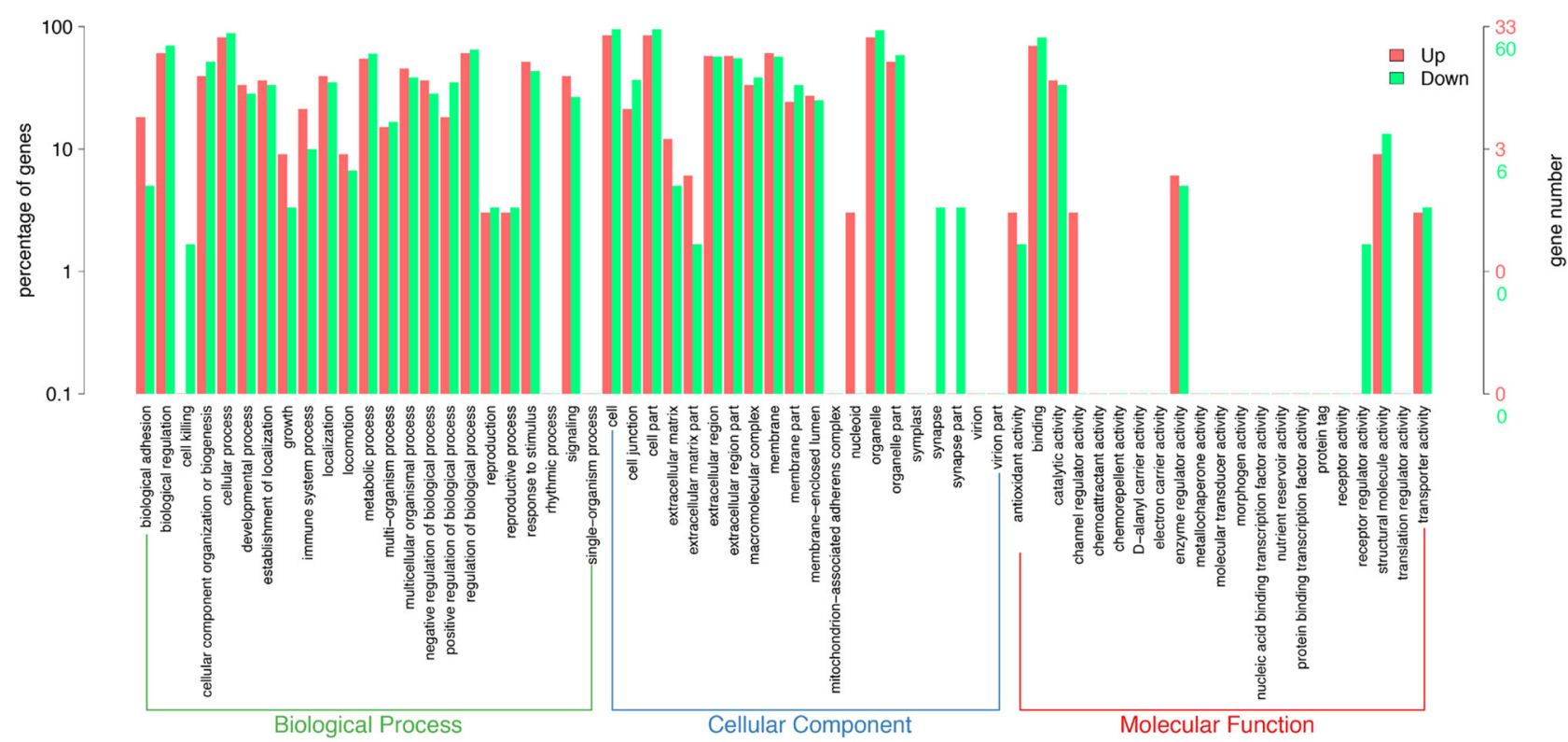

B

Gene Ontology Classification (SDAVF-DV vs STV)

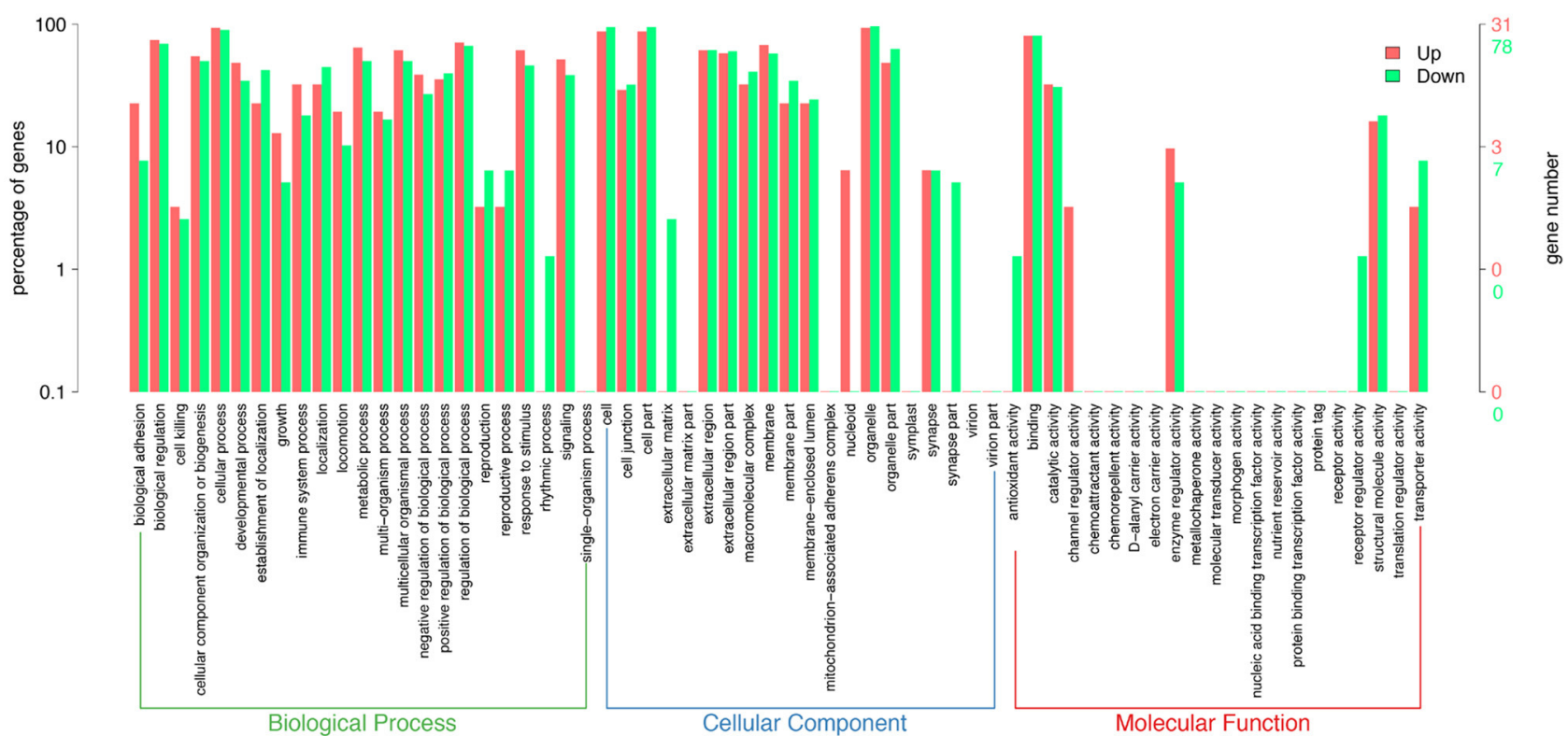

FIGURE 5 | GO analysis of significantly dysregulated proteins and changes in the SDAVF-DV. GO analysis of the up- and downregulated GO terms for the 2.0-fold differentially expressed genes associated with the biological process, cellular component and molecular function categories when comparing the SDAVF-DV with the STA (A) and STV (B). 

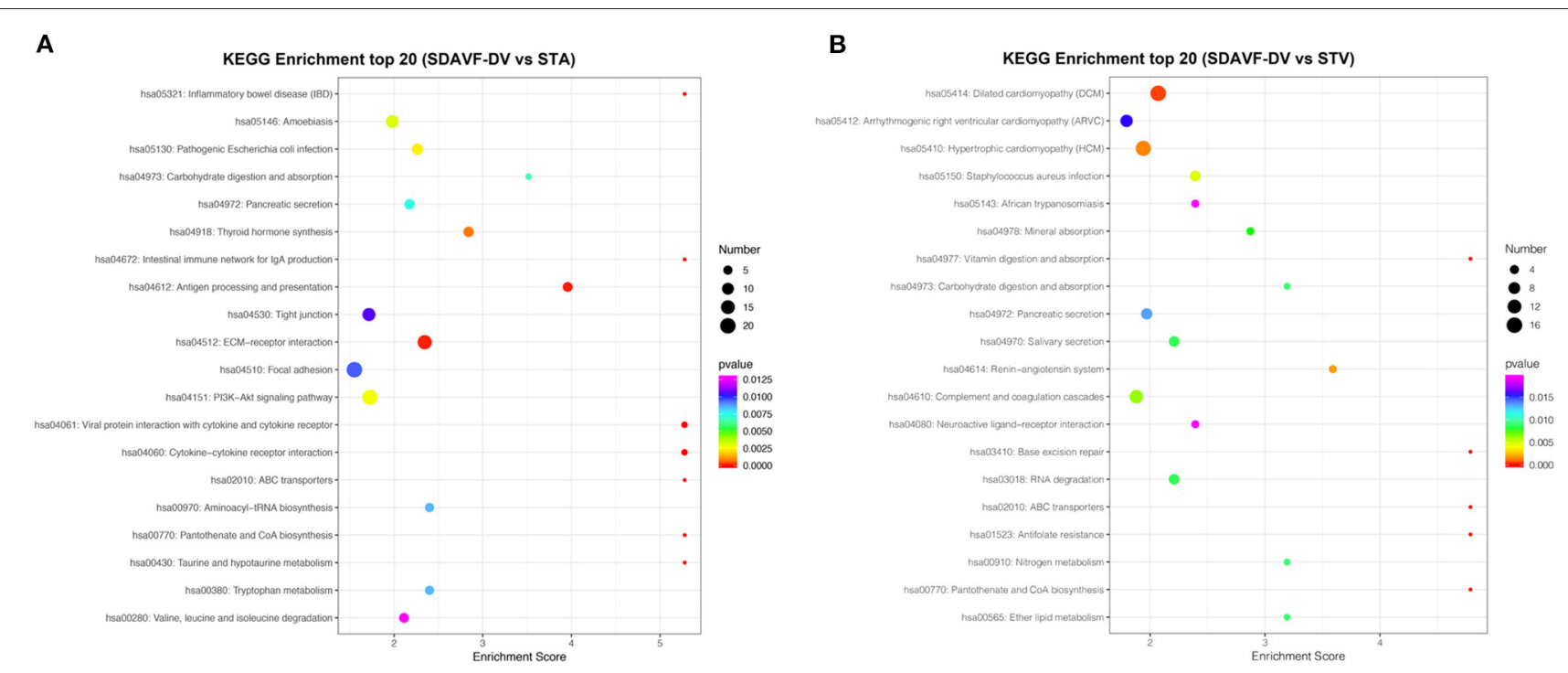

FIGURE 6 | KEGG pathway analysis. The bubble chart shows the KEGG pathway analysis of the top 20 differentially expressed genes when comparing the SDAVF-DV with the STA (A) and STV (B).

When comparing the SDAVF-DV with the STV, and the most differential GO terms expressed in each category were lamellipodium assembly, U6 snRNP, and SH3 domain binding.

The top 10 upregulated and downregulated GO terms based on the comparison of the SDAVF-DV and STV are also listed in Table 2. In GO classification, 93 differentially enriched GO terms were found between the SDAVF-DV and STA: 60 GO terms were upregulated and 33 were downregulated (Figure 5A). Compared with the STV, the SDAVF-DV had 109 differentially enriched GO terms: 31 terms were upregulated and 78 were downregulated (Figure 5B).

\section{Kyoto Encyclopedia of Genes and Genomes Pathway Analysis}

The KEGG pathway analysis of these differentially expressed proteins also demonstrated related pathways. Figure $\mathbf{6}$ shows the number of proteins in each KEGG pathway and the $p$ value of the top 20 pathways. Compared with the STA, the top three differentially expressed protein pathways were focal adhesion, the PI3K-Akt signaling pathway, and the extracellular matrix (ECM)-receptor interaction. Compared with the STV, the top three differentially expressed pathways were dilated cardiomyopathy, hypertrophic cardiomyopathy, and adrenergic signaling in cardiomyocytes.

\section{Protein-Protein Interaction Analysis}

We used the STRING database to analyze the differentially expressed proteins, obtain the interactions/relationships among the differentially expressed proteins, and calculate the combined score. We selected the top 300 proteins and found significant PPIs among them (Figures 7, 8). Compared with the STA and STV, the SDAVF-DV showed upregulated and downregulated proteins, and the top three interaction proteins are listed in Table 3.

\section{DISCUSSION}

SDAVF is a common arteriovenous shunt located inside the dura mater close to the spinal nerve root (8). Venous hypertension, which induces medullar venous outflow disturbances, results in chronic hypoxia and congestive myelopathy (9). The direct intraoperative measurement of the vascular pressure in the fistula can be as high as $74 \%$ of the systemic arterial pressure $(10,11)$. This finding may explain why, in some patients, symptoms become worse during physical activity with a concomitant increase in arterial pressure $(12,13)$. Most previous studies have focused on the histology and anatomy of the SDAVF-DV. Based on intraoperative findings, we demonstrated protein changes in the arterial SDAVF-DV.

Previous studies had revealed that many angiogenetic factors including vascular endothelial growth factor (VEGF), platelet-derived growth factor (PDGF), transforming growth factor (TGF), RAS-MAPK-ERK signal pathway, and PI3K/Akt signal pathway could promote cerebral vascular malformations formation (14). Besides, hyperactivation of oncogenic KRAS in endothelial cells could induce sporadic brain arteriovenous malformations in vivo $(15,16)$. Under long-period venous hypertension, draining vein arterialization begins. There is a growing body of evidence to suggest that angiogenesis plays a role in SDAVF development. The high expression of angiogenic growth factors (especially VEGF) had been proven in human DAVF specimens $(17,18)$. Once sinus thrombosis and venous hypertension are initiated, hypoxia-inducible factor 1 (HIF-1), which was known to be an important upstream regulator of VEGF, was released very rapidly (19). And animal experiments also indicated that release of VEGF was highly related with DAVF formation and that VEGF inhibitor could inhibit its development $(20,21)$. Some genetic changes were proved within arterialized veins. The surgical method enabled drainage vein to be preserved, 


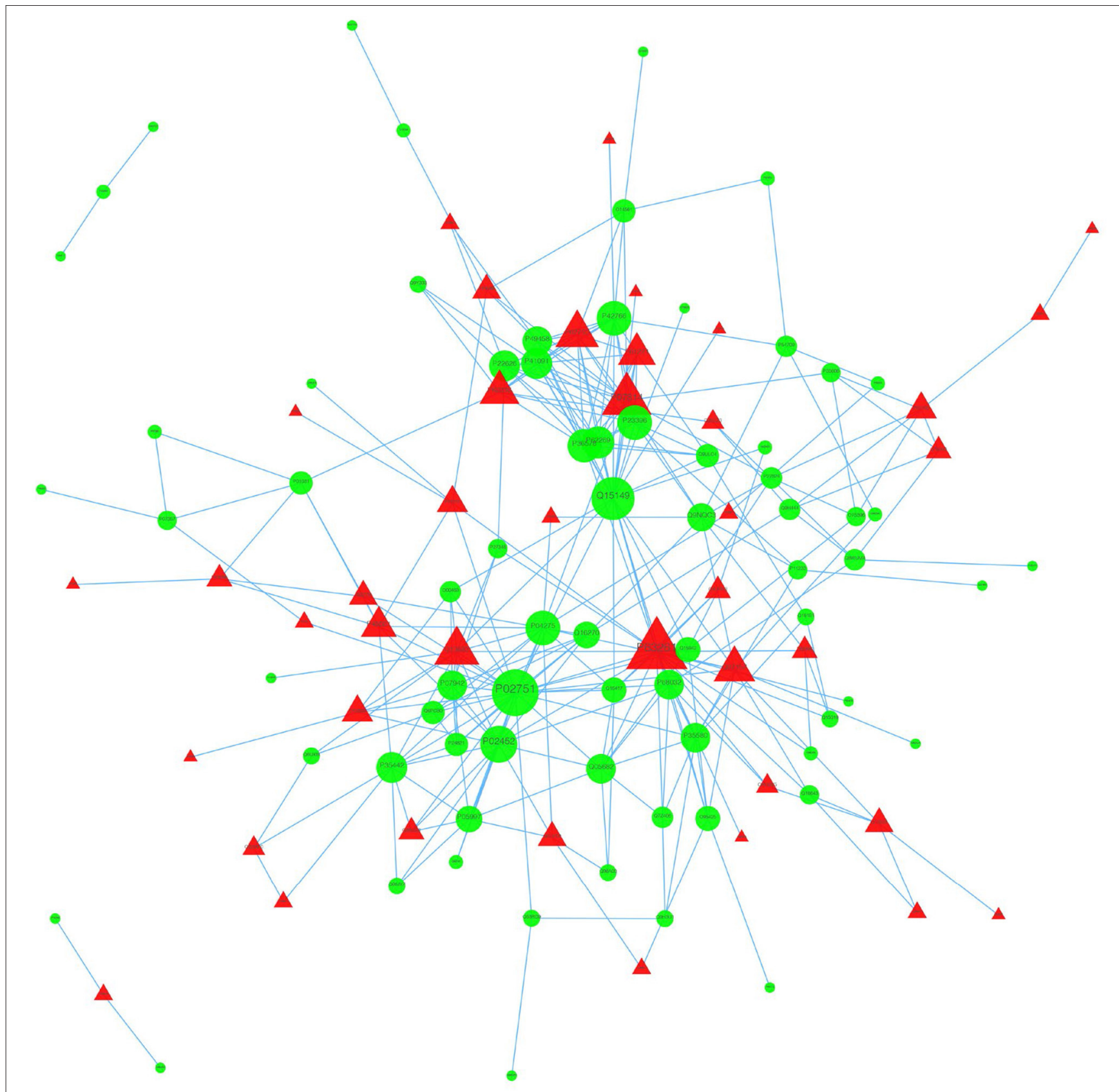

FIGURE 7 | Visualization of PPIs between the SDAVF-DV and STA. Visualization of PPIs for the top 300 proteins using STRING analysis. Red represents upregulated proteins, and green represents downregulated proteins.

and molecular alterations were found in the arterialization of a vein (22).

Pathology analysis revealed thickening of the vascular wall and hyperplasia of the smooth muscle cell layers in the SDAVFDV compared with the STV. In the tunica media, most cells expressed $\alpha$-SMA and AT1, but piezo-1-positive cells were decreased in the SDAVF-DV, suggesting disorder of piezo1 mechanosensitive channels in smooth muscle cells of the
SDAVF-DV. The distribution of the ECM showed a special vascular structure under sustained high vascular pressure.

A clear understanding of the mechanism of SDAVF development is still lacking. Our study was the first to perform a comparative proteome analysis and show the differential expression of proteins in arterialized SDAVFDVs compared with normal arteries and veins. In general, most of the proteins were the same between the three 


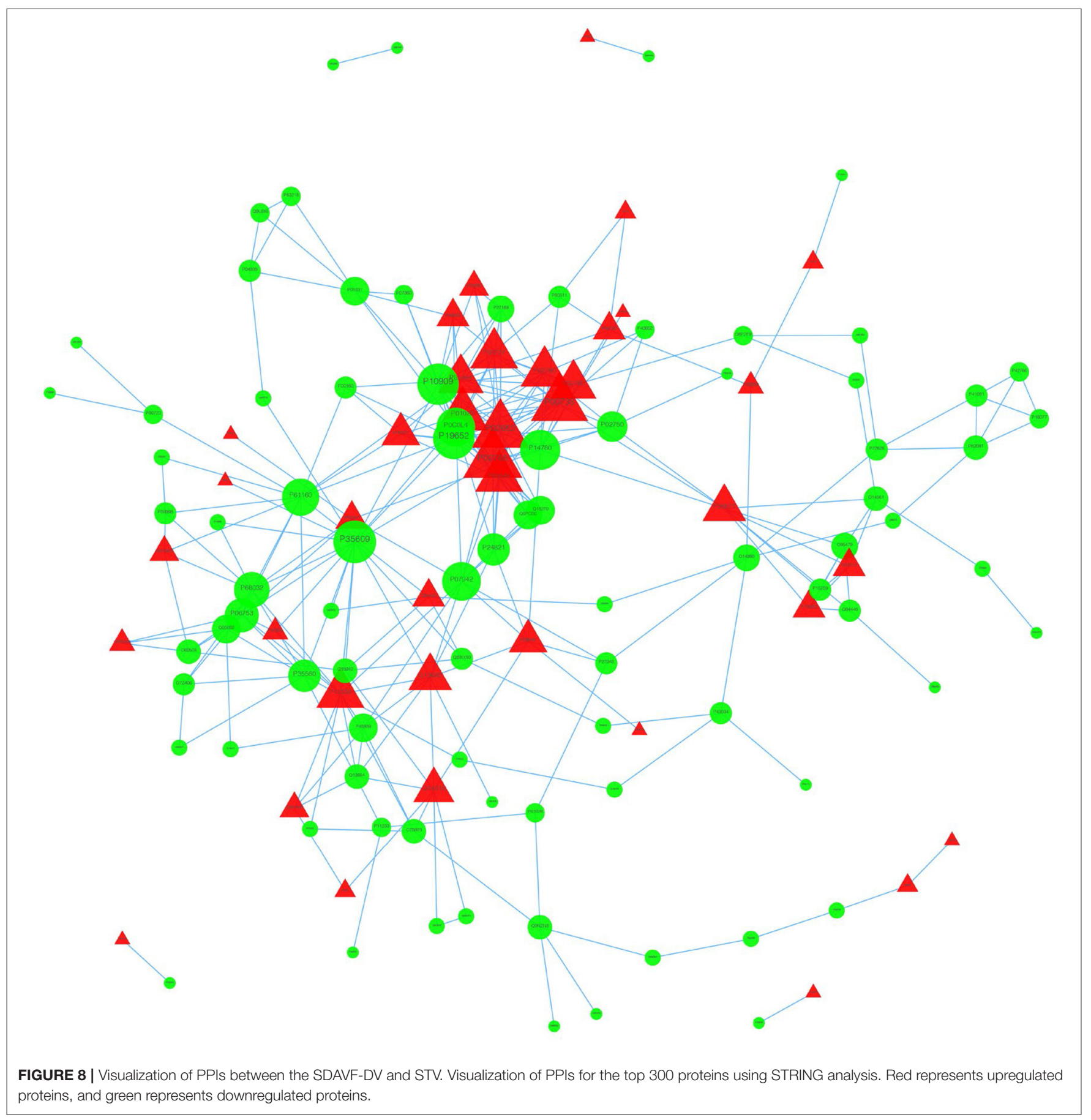

groups. Because of its special pathophysiology, the SDAVFDV showed specific protein expression compared with the STA and STV.

In the intraoperative observation, the SDAVF-DV showed arterial morphology. Thron proposed a hypothesis based on spine arteriovenous shunt anatomy (13). Takai showed that the vessel wall of the proximal subarachnoid portion of the intradural draining vessels was irregularly thickened by collagen, exhibited elastic fibrosis, and was without a continuous internal elastic lamina and a regular smooth muscle layer. The diameter of the vessels was significantly enlarged (23).

After GO analysis, the SDAVF-DV showed a decrease in smooth muscle contractile fibers, which might indicate smooth muscle cell dysfunction. This might be induced by long-range venous hypertension stretching on the SDAVFDV. Stretch plays an important role in maintaining smooth muscle cell function and regulating inflammation. A former study showed that mechanical stretch-induced endoplasmic 
TABLE 3 | Top three interaction protein number proteins

\begin{tabular}{lll}
\hline & Gene ID & Upregulated protein \\
\hline SDAVF-DV vs. STA & & \\
Upregulated & P63261 & Actin, cytoplasmic 2 \\
& P07814 & Bifunctional glutamate/proline-tRNA ligase \\
& P62750 & 60S ribosomal protein L23a \\
P02751 & Fibronectin \\
Downregulated & Q15149 & Plectin \\
& P02452 & Collagen alpha-1(I) chain \\
SDAVF-DV vs STV & & \\
Upregulated & P00738 & Haptoglobin \\
& P02652 & Apolipoprotein A-II \\
& P02765 & Alpha-2-HS-glycoprotein \\
& P19652 & Alpha-1-acid glycoprotein 2 \\
P10909 & Clusterin \\
Downregulated & P14780 & Matrix metalloproteinase-9 \\
& & \\
\hline
\end{tabular}

reticulum stress, apoptosis, and inflammation contribute to thoracic aortic aneurysm and dissection (24). In our research, we also identified that mechanical stretching could induce smooth muscle cell changes from a contract phenotype to an inflammatory phenotype $(25,26)$. The regulation of inflammatory factors is related to the hypothesis on SDAVF formation. In THE KEGG and PPI analyses, the ECM and focal adhesion showed obvious changes. Degeneration of the ECM is primarily induced by the secretion of inflammatory cytokines and cell infiltration in cerebral vascular disease (27-29). During SDAVF formation, inner vessel wall inflammation might contribute to an insufficient ECM and trigger changes in pathological proteins.

\section{CONCLUSIONS}

To our knowledge, few studies have focused on the SDAVF-DV. However, several researchers have investigated its pathological characteristics $(23,29)$. We first examined protein changes to determine whether the lesion vessel was an artery, vein, or veinto-artery transition.

In this study, we found the vessel wall of SDAVFDV remodeling, which is different with artery and vein

\section{REFERENCES}

1. Amanieu C, Hermier M, Peyron N, Chabrol A, Deiana G, Manera L. Spinal dural arteriovenous fistula. Diagn Interv Imaging. (2014) 95:897-902. doi: 10.1016/j.diii.2013. 08.007

2. Krings T, Geibprasert S. Spinal dural arteriovenous fistulas. AJNR Am J Neuroradiol. (2009) 30:639-48. doi: 10.3174/ajnr.A1485

3. Hasan D, Hashimoto T, Kung D, Macdonald RL, Winn HR, Heistad D. Upregulation of cyclooxygenase-2 (COX-2) and microsomal prostaglandin E2 synthase-1 (mPGES-1) in wall of ruptured histologically even it is similar to artery in morphology. The results of proteomics showed smooth muscle cell dysfunction and inflammation changes of SDAVF-DV. Our study adds new information on the formation of SDAVF to the realm of protein changes in the draining vein using proteomics. This finding may shed light on the mechanism of SDAVF formation.

\section{DATA AVAILABILITY STATEMENT}

The raw data supporting the conclusions of this article will be made available by the authors, without undue reservation.

\section{ETHICS STATEMENT}

The studies involving human participants were reviewed and approved by Ethics Committee of Huashan Hospital, Fudan University. The patients/participants provided their written informed consent to participate in this study.

\section{AUTHOR CONTRIBUTIONS}

PL: conception and design of study, provision of study materials, collection and/or assembly of data, data analysis and interpretation, manuscript writing, and final approval of manuscript. YS: data analysis and interpretation, and manuscript writing. SL and YL: database input and data interpretation. YZ: data analysis. YS: manuscript writing. WZ: provision of study and revision and final approval of manuscript. QA: conception and design of study and revision and final approval of manuscript. WZ and QA: are co-corresponding authors of this article. All authors contributed to the article and approved the submitted version.

\section{FUNDING}

The Outstanding Academic Leaders Program of Shanghai Municipal Commission of Health and Family Planning (No. 2017BR006 to WZ); National Natural Science Foundation of China (No. 81571102, No. 81870911 to WZ; No. 81801148 to PL). This study was supported by Clinical Research Plan of SHDC (No. SHDC2020CR2034B to WZ, No. SHDC2020CR4033 to $\mathrm{KQ})$. human cerebral aneurysms: preliminary results. Stroke. (2012) 43:1964-7. doi: 10.1161/STROKEAHA.112.655829

4. Wang J, Yu L, Huang X, Wang Y, Zhao J. Comparative proteome analysis of saccular intracranial aneurysms with iTRAQ quantitative proteomics. $J$ Proteomics. (2016) 130:120-8. doi: 10.1016/j.jprot.2015.09.014

5. Neilson KA, Ali NA, Muralidharan S, et al. Less label, more free: approaches in label-free quantitative mass spectrometry. Proteomics. (2011) 11:53553. doi: $10.1002 /$ pmic. 201000553

6. Zhuang $\mathrm{Y}, \mathrm{Ma} \mathrm{F}, \mathrm{Li}-\mathrm{Ling} \mathrm{J}, \mathrm{Xu} \mathrm{X}, \mathrm{Li} \mathrm{Y}$. Comparative analysis of amino acid usage and protein length distribution between alternatively and non-alternatively spliced genes across six eukaryotic 
genomes. Mol Biol Evol. (2003) 20:1978-85. doi: 10.1093/molbev/ms g203

7. Sun $\mathrm{X}$, Zhang H, Luo L, et al. Comparative proteomic profiling identifies potential prognostic factors for human clear cell renal cell carcinoma. Oncol Rep. (2016) 36:3131-8. doi: 10.3892/or.2016.5159

8. Kendall BE, Logue V. Spinal epidural angiomatous malformations draining into intrathecal veins. Neuroradiology. (1977) 13:1819. doi: 10.1007/BF00344211

9. Thron A, Krings T, Otto J, Mull M, Schroeder JM. The transdural course of radicular spinal cord veins-a microangiographical and microscopical study. Clin Neuroradiol. (2015) 25:361-9. doi: 10.1007/s00062-015-0476-x

10. Hassler W, Thron A. Flow velocity and pressure measurements in spinal dural arteriovenous fistulas. Neurosurg Rev. (1994) 17:29-36. doi: 10.1007/BF00309983

11. Hassler W, Thron A, Grote EH. Hemodynamics of spinal dural arteriovenous fistulas. An intraoperative study J Neurosurg. (1989) 70:360-70. doi: 10.3171/jns.1989.70.3.0360

12. Aminoff MJ, Barnard RO, Logue V. The pathophysiology of spinal vascular malformations. J Neurol Sci. (1974) 23:25563. doi: 10.1016/0022-510x(74)90229-9

13. Khurana VG, Perez-Terzic CM, Petersen RC, Krauss WE. Singing paraplegia: a distinctive manifestation of a spinal dural arteriovenous fistula. Neurology. (2002) 58:1279-81. doi: 10.1212/wnl.58.8.1279

14. Barbosa Do Prado L, Han C, Oh SP, Su H. Recent advances in basic research for brain arteriovenous malformation. Int J Mol Sci. (2019) 20:5324. doi: 10.3390/ijms20215324

15. Park ES, Kim S, Huang S, Yoo JY, Körbelin J, Lee TJ, et al. Selective endothelial hyperactivation of oncogenic kras induces brain arteriovenous malformations in mice. Ann Neurol. (2021) 89:926-41. doi: 10.1002/ana.26059

16. Fish JE, Flores Suarez CP, Boudreau E, Herman AM, Gutierrez MC, Gustafson $\mathrm{D}$, et al. Somatic gain of KRAS function in the endothelium is sufficient to cause vascular malformations that require MEK but not PI3K signaling. Circ Res. (2020) 127:727-43. doi: 10.1161/CIRCRESAHA.119.316500

17. Tirakotai W, Bertalanffy H, Liu-Guan B, Farhoud A, Sure U. Immunohistochemical study in dural arteriovenous fistulas and possible role of local hypoxia for the de novo formation of dural arteriovenous fistulas. Clin Neurol Neurosurg. (2005) 107:455-60. doi: 10.1016/j.clineuro.2004.11.014

18. Uranishi R, Nakase $H$, Sakaki $T$. Expression of angiogenic growth factors in dural arteriovenous fistula. J Neurosurg. (1999) 91:781-6. doi: 10.3171/jns.1999.91.5.0781

19. Zhu Y, Lawton MT, Du R, Shwe Y, Chen Y, Shen F, et al. Expression of hypoxia-inducible factor-1 and vascular endothelial growth factor in response to venous hypertension. Neurosurgery. (2006) 59:687-96. doi: 10.1227/01

20. Bhogal P, Yeo LL, Henkes H, Krings T, Söderman M. The role of angiogenesis in dural arteriovenous fistulae: the story so far. Interv Neuroradiol. (2018) 24:450-4. doi: 10.1177/1591019918764090

21. Li Q, Zhang Q, Huang QH, Fang YB, Zhang ZL, Xu Y, et al. A pivotal role of the vascular endothelial growth factor signaling pathway in the formation of venous hypertension-induced dural arteriovenous fistulas. Mol Med Rep. (2014) 9:1551-8. doi: 10.3892/mmr.2014.2037
22. Martinez L, Tabbara M, Duque JC, Selman G, Falcon NS, Paez A, et al. Transcriptomics of human arteriovenous fistula failure: genes associated with nonmaturation. Am J kidney Dis Off J Natl Kidney Found. (2019) 74:7381. doi: 10.1053/j.ajkd.2018.12.035

23. Takai K, Komori $\mathrm{T}$, Taniguchi M. Microvascular anatomy of spinal dural arteriovenous fistulas: arteriovenous connections and their relationships with the dura mater. J Neurosurg Spine. (2015) 23:526-33. doi: 10.3171/2014.11.SPINE14786

24. Jia L-X, Zhang W-M, Zhang H-J, Li T-T, Wang Y-L, Qin Y-W, et al. Mechanical stretch-induced endoplasmic reticulum stress, apoptosis and inflammation contribute to thoracic aortic aneurysm and dissection. J Pathol. (2015) 236:373-83. doi: 10.1002/path.4534

25. Liu P, Song Y, Zhou Y, Liu Y, Qiu T, An Q, et al. Cyclic Mechanical stretch induced smooth muscle cell changes in cerebral aneurysm progress by reducing collagen type IV and collagen type VI levels. Cell Physiol Biochem Int J Exp Cell Physiol Biochem Pharmacol. (2018) 45:105160. doi: 10.1159/000487347

26. Liu P, Shi Y, Fan Z, Zhou Y, Song Y, Liu Y, et al. Inflammatory Smooth muscle cells induce endothelial cell alterations to influence cerebral aneurysm progression via regulation of integrin and VEGF expression. Cell Transplant. (2019) 28:713-22. doi: 10.1177/0963689718815824

27. Chalouhi N, Ali MS, Jabbour PM, Tjoumakaris SI, Gonzalez LF, Rosenwasser $\mathrm{RH}$, et al. Biology of intracranial aneurysms: role of inflammation. J Cereb blood flow Metab Off J Int Soc Cereb Blood Flow Metab. (2012) 32:165976. doi: $10.1038 /$ jcbfm.2012.84

28. Starke RM, Raper DMS, Ding D, Chalouhi N, Owens GK, Hasan DM, et al. Tumor necrosis factor- $\alpha$ modulates cerebral aneurysm formation and rupture. Transl Stroke Res. (2014) 5:269-77. doi: 10.1007/s12975-013-0287-9

29. Benhaiem N, Poirier J, Hurth M. Arteriovenous fistulae of the meninges draining into the spinal veins. A histological study of 28 cases. Acta Neuropathol. (1983) 62:103-11. doi: 10.1007/BF00684926

Conflict of Interest: The authors declare that the research was conducted in the absence of any commercial or financial relationships that could be construed as a potential conflict of interest.

Publisher's Note: All claims expressed in this article are solely those of the authors and do not necessarily represent those of their affiliated organizations, or those of the publisher, the editors and the reviewers. Any product that may be evaluated in this article, or claim that may be made by its manufacturer, is not guaranteed or endorsed by the publisher.

Copyright (c) $2021 \mathrm{Liu}$, Shi, Li, Liu, Zhou, Song, Zhu and An. This is an open-access article distributed under the terms of the Creative Commons Attribution License (CC $B Y)$. The use, distribution or reproduction in other forums is permitted, provided the original author(s) and the copyright owner(s) are credited and that the original publication in this journal is cited, in accordance with accepted academic practice. No use, distribution or reproduction is permitted which does not comply with these terms. 